\title{
WALLET KEY MANAGEMENT IN BLOCKCHAIN TECHNOLOGY
}

\author{
Dominic BUCERZAN \\ "Aurel Vlaicu” University of Arad, Romania \\ dominic@bbcomputer.ro \\ Crina Anina BEJAN \\ "Aurel Vlaicu” University of Arad, Romania \\ ratiu_anina@yahoo.com
}

\begin{abstract}
Cryptocurrency is a technology shift that spread all over the world and improved our economic and social interactions. Specialist in the field admit that it is a phenomenon at its early phase and at this time, long term predictions regarding its evolution and effects cannot be accurate. Blockchain is the layer on which crypto currency rely. Although it is considered to be the most secure digital system, lately events showed that its applicability to different fields can make it vulnerable to some types of threats that can create losses of information, values and money. In this paper we identify some of this threats and vulnerabilities. We choose to focus on crypto wallets vulnerabilities and propose a method to improve its security by using steganography to optimize private key storage.
\end{abstract}

Keywords: Blockchain, crypto currency, crypto wallets, private key.

JEL classification: J11, C8, L86.

DOI: $10.24818 / \mathrm{ie} 2020.02 .05$

\section{Introduction}

Computer science, mathematics, cryptography and economics converge to form the roots of blockchain technology, being the propulsion for the second era of digital economy [1].

Figure 1 [2] shows the evolution of blockchain technology.

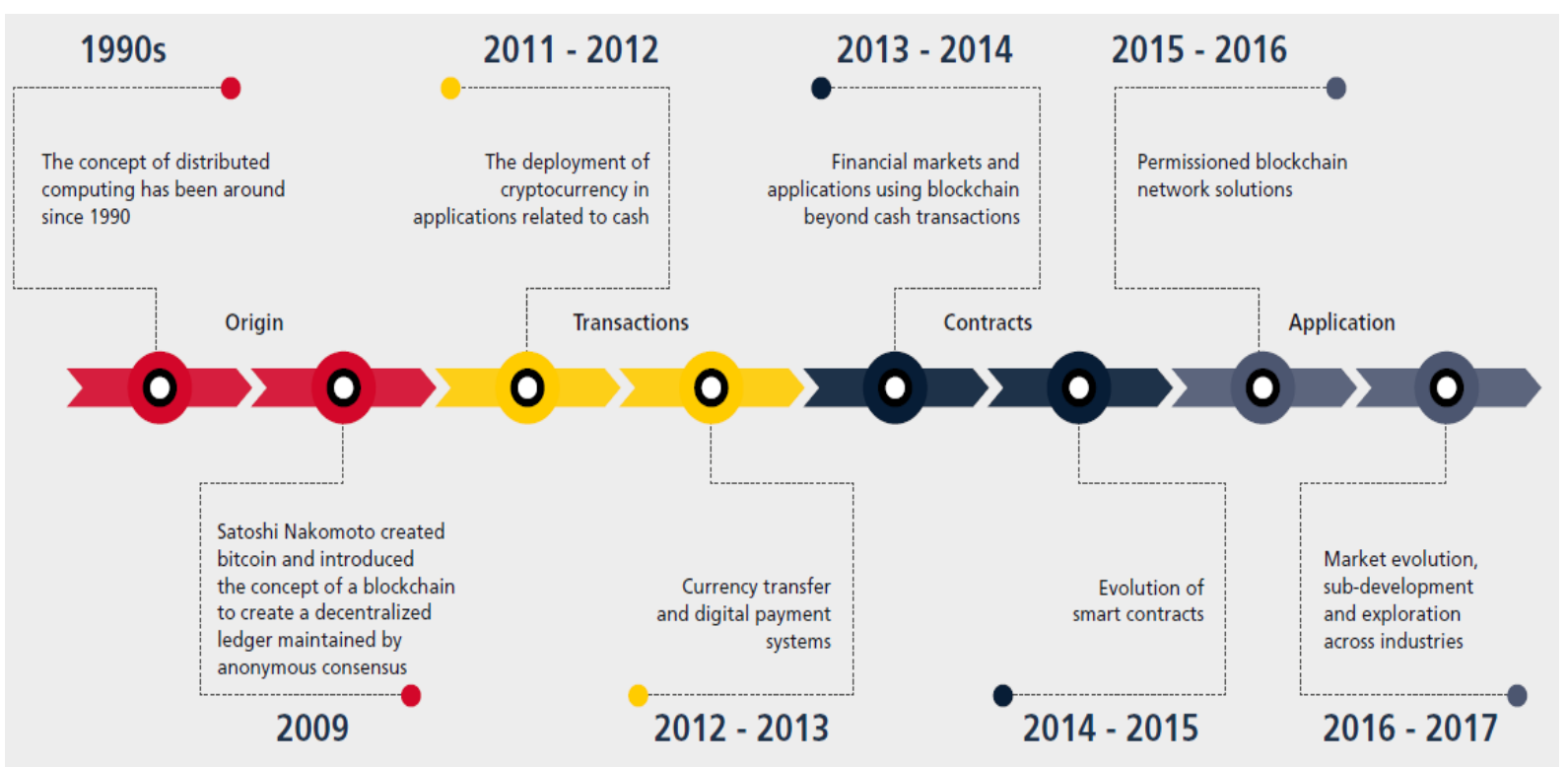

Figure 1. Evolution of Blockchain technology 
Blockchain started back in 2008 merging in an original manner technology that had existed before. It offers at a global scale trust and collaboration. Using protocols, cryptography and computer code [3], it strengthens trust, collaboration and communication between users all over the world without involving a third-party authority.

A blockchain system involves the fallowing components (see figure 2) [4]:

- Digital Ledger: stores the transactions. It is public and can be read by every user. Only miners can write to the ledger. It is immutable, once a transaction is written to the ledger it cannot be modified afterwards;

- Peer Network: the network of miners which maintains the ledger. A copy of the ledger is kept by each node. The nodes from the network use a consensus protocol in order to agree on every transaction that is written to the ledger;

- Membership Services: manages user identity;

- Smart Contract: programs and data that run on the blockchain;

- Wallet: tracks user associated digital assets and stores users' credentials;

- Events: updates and actions on the blockchain;

- Systems Management: allows to manage the blockchain components;

- Systems Integration: allows the blockchain to be integrated with external systems.

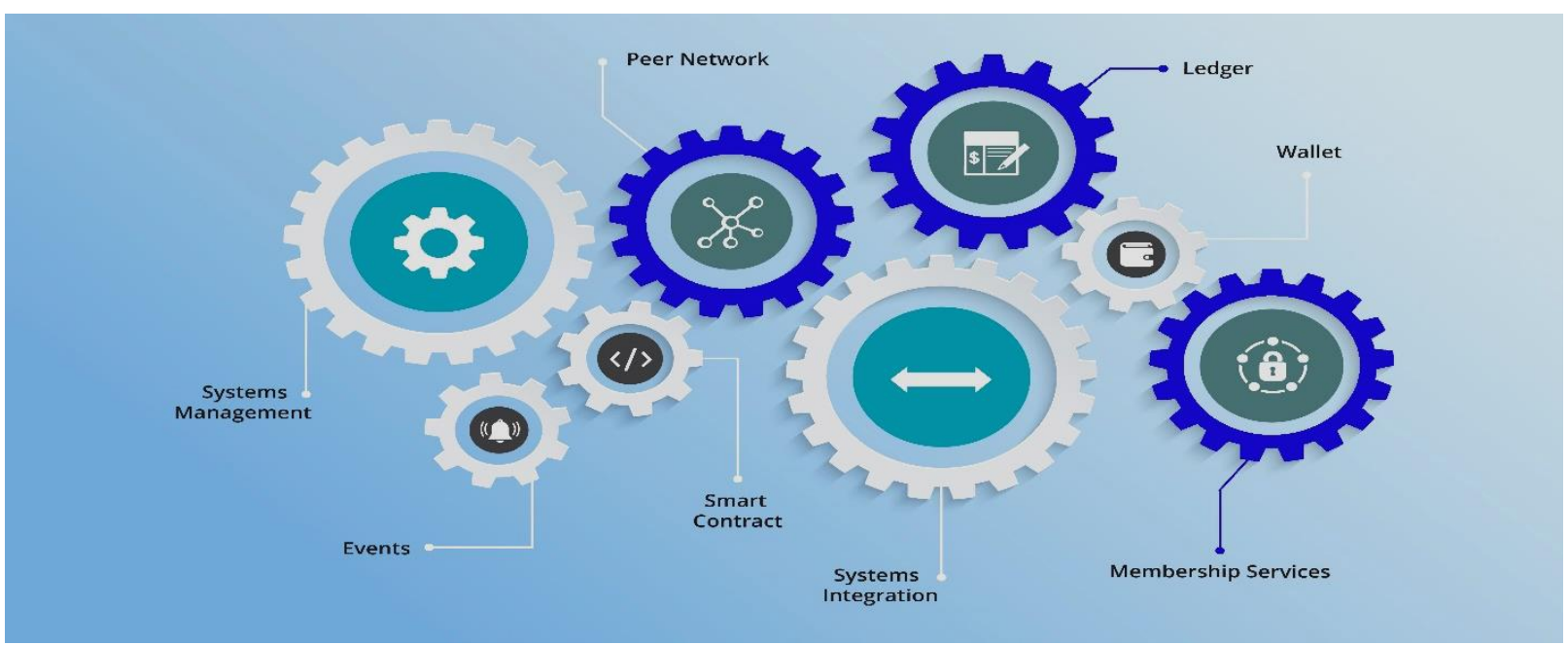

Figure. 2. Blockchain components [4]

\section{Cryptocurrency}

Cryptocurrency is the first application of blockchain. Primarily design for bitcoin, blockchain system have become the layer base for several cryptocurrencies. According to [5] today there are more than 5200 traded cryptocurrencies with a market capital of over 155 billion of EUR. Figure 3 shows the evolution of cryptocurrency market [5]. 


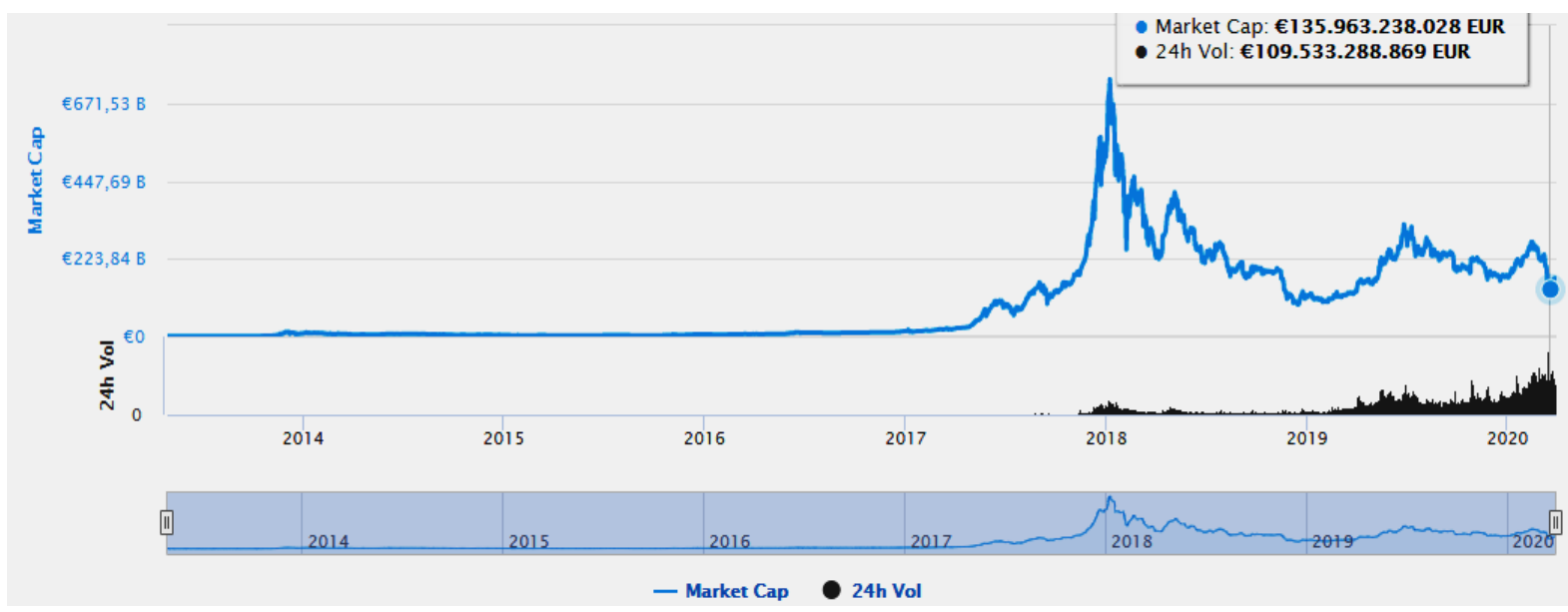

Figure 3. Total market capitalization of cryptocurrencies [5]

From all cryptocurrencies, bitcoin remains for more than 12 years the most transacted one, having a market capital of more than $64 \%$ (see figure 4) [5].

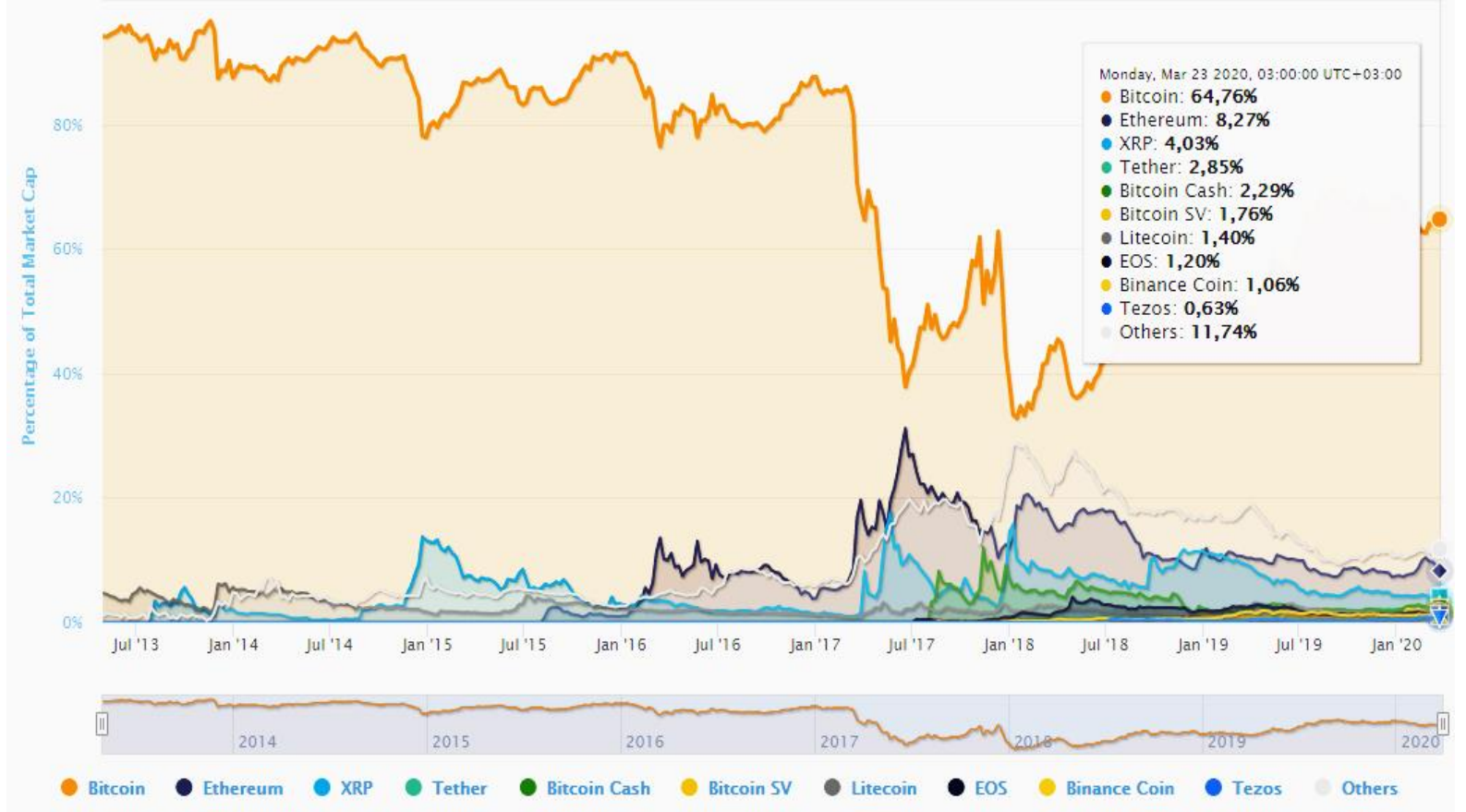

Figure 4. Bitcoin Market Capital

To use cryptocurrencies, one does not have to be a full node miner. Like in paper currency to use money, one does not have to be a bank. Usual cryptocurrency users do not need to download the entire blockchain or to have expensive and complex technological devices. They just have to use a cryptocurrency wallet. This type of devices is explained in section three of this paper.

\section{Cryptocurrency Wallets}

A wallet is used to store the identity of a user, by which the user can easily authenticate ownership of digital assets and manage transactions on the blockchain.

Cryptocurrency wallets come in mammy forms. According to [6], based on their connectivity to internet, wallets can be classified in two main categories: 
- hot wallets - are connected to the internet. They include: online web wallets and Smartphone apps wallets.

- cold wallets - stay offline. They include: paper wallets, hardware wallets and brain wallets. Technically wallets use Public Key Infrastructure (PKI) to ensure one's identity. To manage wallet keys authors, propose various solutions [7-10]. Every type of wallets presents its own particularities and security vulnerabilities like[6,7]:

- hot wallets are always connected to the internet, being exposed permanently to cyberattacks. Also, another threat in this case arose when the key are stored on a device which is connected to the internet. If an attacker stole the user private key it has access to the user digital assets;

- paper wallets are literally a piece of paper with the public key printed on it. When using this type of wallets, the user might face some attackers that try to steal the key using the printer which will print the paper or compromising the operation system that runs on the computer used to print the wallet. If the user loses the paper or by some manners the paper gets destroyed the owner could not get in the possession of its cryptocurrency;

- hardware wallets are small devices that plug into computer or smartphone to sign the transaction. If the user loses the device or it gets stolen it doesn't have any possibility to recover owned money;

- brain wallets are considered to be the secures of all because it supposes that the user memorizes the private key. It offers the possibility to memorize a collection of words or phrases to have a more easily memorizable set of characters than the users straight up private key. This type of wallets has to face the fact that humans aren't as random as a computer, so the words someone chooses for their brain wallet might easily be guessed such an attack is called a dictionary attack.

There are many blockchain based PKI approaches. In the end users have to tradeoff between convenience and security. In [7] authors present a classification of certain criteria which can be used to delimit the advantages and disadvantages of present wallets (see Figure 5).

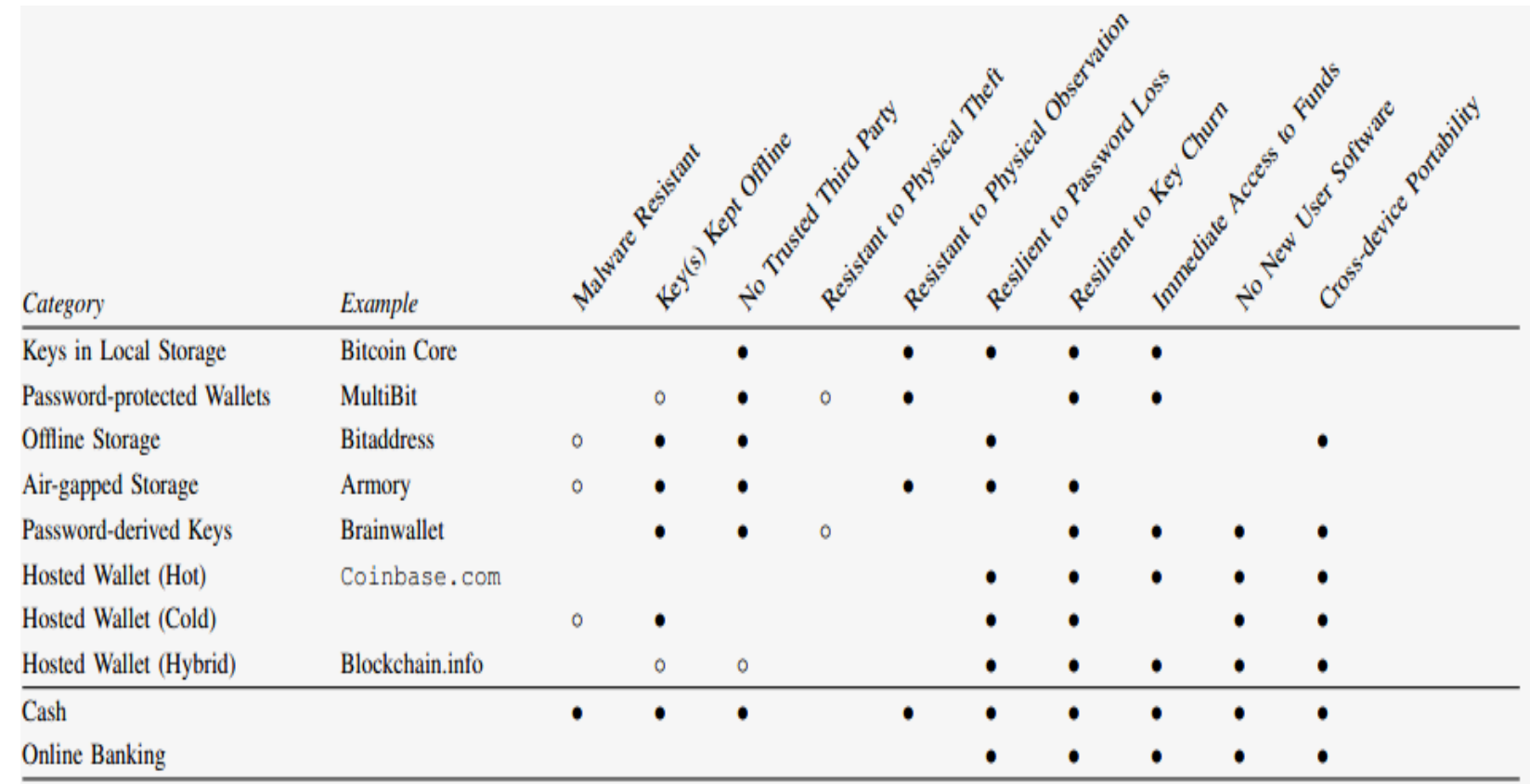

Figure 5. A comparison of key management solutions for crypto wallets, • indicates

The category of client is awarded the benefit in the corresponding column, o partially awards the benefit [7] 
A way to optimize the security of crypto-wallets is by using steganography for storing the private keys [11]. Steganography hides a "secret information" in different carriers in such a way that it becomes difficult to detect. Commonly the carriers are media files (like images, audio, video).

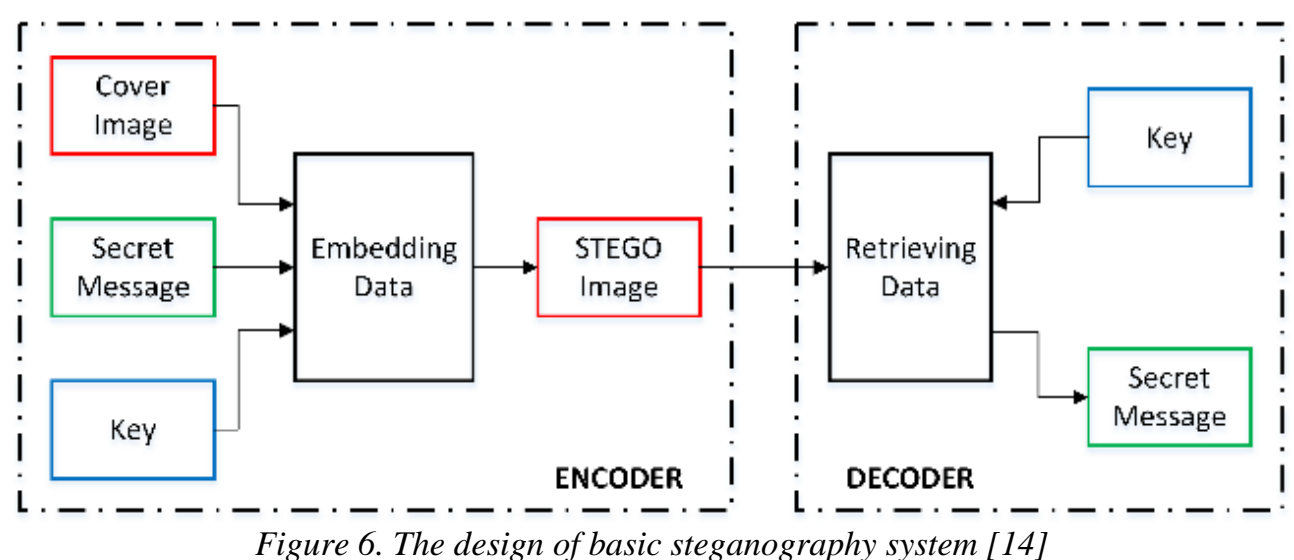

This technique suppose that the private key of a user is hidden in a media file which can be store on different devices (computers, smart devices). When the user wants to connect to the wallet, the media file which holts the key is selected, the key is automatically extracted and the user is logged to the wallet.

In this paper we propose a new solution to store the private key by using digital steganography combined with cryptography, namely SmartSteg. The method is described in the next section of the paper.

\section{SmartSteg-wallet}

SmartSteg allows to secretly transferring different types of data using different types of devices. The solution is based on secret key stream chipper cryptography combined with Last Significant Bit (LSB) steganography optimized by a pseudo random selection function $[12,13]$. SmartSteg provides two layers of security for the secret data. First the secret information is encrypted using a proprietary algorithm. Second the encrypted information is embedded in the cover file using an improved LSB algorithm, based on the pseudo random selection function. SmartSteg adds another layer of security to the proposed architecture by processing the entire surface of the cover image in order to conceal the length of the secret file [11].

The proposed steganographic solution has been tested on several devices (windows devices and Android smart devices) and it proved high computational and security performances. It is a high-speed solution, the process of hiding and revealing the secret information takes some nanosecond which are unnoticeable for the human perspective.

The proposed solution is still in its design stage and it fallows to be implemented using a private wallet software for the measurement needed to be taken in order to establish its performances for blockchain technology.

\section{Conclusions}

In this paper we propose a new solution to enhance the security of cryptocurrency wallets PKI management. It is based on SmartSteg technique which combines cryptography and steganography. The solution is still a prototype and has to be implemented for blockchain systems. 
www.conferenceie.ase.ro

\section{References}

[1] D. Bucerzan and C. Bejan, "On the Cryptoeconomics Emerging Research Areas", in Proceedings of the 18th International Conference on INFORMATICS in ECONOMY, 2018, pp. 273-278., DOI:10.12948/ie2019.04.19.

[2] ***, "Blockchain in Logistics. Perspectives on the upcoming impact of blockchain technology and use cases for the logistics industry", DHL Customer Solutions \& Innovation, 2018

[3] Colchester, J. (2018), Blockchain: an Overview, A Complexity Labs Publication. Retrieved January 19, 2018, from http://complexitylabs.io/blockchain-book/.

[4] ***, "Blockchain: Understanding Its Uses and Implications", LinuxFoundationX: LFS170x, Retrieved on August 2018 at: https://courses.edx.org/courses/course-v1

[5] ***, Blockchain Explorer, Retrived on March 2020 at: https://coinmarketcap.com/charts/.

[6] ***, Bitcoin and Cryptocurrencies, BerkeleyX: CS198.1x, Retrieved on March 2020 at: https://courses.edx.org/courses/course-v1:BerkeleyX+CS198.1x+3T2019/course/

[7] S. Eskandari, J. Clark, D. Barrera and E. Stobert, (2018). A first look at the usability of bitcoin key management. arXiv preprint arXiv:1802.04351, Retrieved on March 2020 at: https://arxiv.org/pdf/1802.04351.pdf

[8] J. Brito, and A. Castillo, Bitcoin: A primer for policymakers Mercatus Center at George Mason University, 2013.

[9] ***, Blockchain Tech Beyond Bitcoin, Sutardja Center for Entrepreneurship \& Technology Technical Report, 2015, Retrieved on March 2020 at: https://scet.berkeley.edu/wpcontent/uploads/BlockchainPaper.pdf

[10] O. Pal, B. Alam, V. Thakur and S. Singh, (2019). "Key management for blockchain technology". ICT Express.

[11] D. Bucerzan, C. Raţiu and M. J. Manolescu (2013). "SmartSteg: a New Android Based Steganogrphy Application". In: International Journal of Computers Communications I\& Control, vol. 8(5), pp. 681-688.

[12] D. Bucerzan and C. Raţiu (2015). "Contributions to Steganographic Techniques on Mobile Devices”. In: Bica I., Naccache D., Simion E. (eds) Innovative Security Solutions for Information Technology and Communications. SECITC 2015. Lecture Notes in Computer Science, vol 9522. Springer, Cham.

[13] D. Bucerzan and C. Raţiu, "Testing methods for the efficiency of modern steganography solutions for mobile platforms," 2016 6th International Conference on Computers Communications and Control (ICCCC), Oradea, 2016, pp. 30-36.

[14] D. Quang and K. Insoo. (2017). FPGA implementation of LSB-based steganography. 15. 151-159. 10.6109/jicce.2017.15.3.151. 